\title{
Vólvulo del ciego, una entidad infrecuente como causa de abdomen agudo en embarazadas: reporte de caso clínico y revisión de la literatura
}

\author{
Volvulus of the cecum as an uncommon cause of acute abdomen in \\ pregnant women: Case report and review of the literature
}

\begin{abstract}
Mauricio Pedraza-Ciro ${ }^{1} \mathbb{D}$, Juan Guillermo $\operatorname{Ramos}^{2} \mathbb{D}$, María Cristina Geney ${ }^{3} \mathbb{D}$, María Daniela Moreno ${ }^{4} \mathbb{D}$, Marcia Roxana Santafe ${ }^{4} \mathbb{D}$, Paula Vega ${ }^{3} \mathbb{D}$, Diana Puerto ${ }^{3} \mathbb{D}$, Jorge E. Niño ${ }^{3} \mathbb{D}$
\end{abstract}

\begin{abstract}
Médico, especialista en Cirugía General, Hospital Universitario Clínica San Rafael, Bogotá, D.C., Colombia.
Médico, especialista en Ginecología y Obstetricia, Hospital Universitario Clínica San Rafael, Bogotá, D.C., Colombia.

Médico, especialista en Cirugía General, Departamento cirugía general, Universidad El Bosque, Bogotá, D.C., Colombia.

Grupo de investigación en cirugía general y subespecialidades (GICS), Universidad El Bosque, Bogotá, D.C., Colombia.
\end{abstract}

\section{Resumen}

Introducción. El vólvulo cecal en el embarazo es una patología infrecuente a nivel mundial, con un espectro clínico variado y limitación en estudios diagnósticos, lo que puede resultar en un desenlace ominoso.

Caso clínico. Se presenta el caso de una gestante en segundo trimestre del embarazo quien presenta abdomen agudo secundario a vólvulo cecal y sufrimiento fetal. La paciente fue llevada a cesárea de urgencia, donde además se practicó cecectomía y anastomosis íleo transversa, con mortalidad fetal.

Discusión. El vólvulo cecal forma parte de los diagnósticos diferenciales en caso de abdomen agudo durante el embarazo. Tiene un pronóstico reservado, en donde pueden presentarse desenlaces maternos y perinatales adversos, con mortalidad maternofetal.

Palabras clave: vólvulo intestinal; ciego; abdomen agudo; embarazo; complicaciones del embarazo; resultado del embarazo.

Fecha de recibido: 30/09/2019 - Fecha de aceptación: 06/02/2020

Correspondencia: Mauricio Pedraza Ciro, Carrera 72 \# 181-55, Bogotá, D.C., Colombia. Teléfono: 3013709557

Correo electrónico: mpedraza93@gmail.com

Citar como: Pedraza-Ciro M, Ramos JG, Geney MC, Moreno MD, Santafe MR, Vega P, et al. Vólvulo del ciego, una entidad infrecuente como causa de abdomen agudo en embarazadas: Reporte de caso clínico y revisión de la literatura. Rev Colomb Cir. 2020;35:699-704. https://doi.org/10.30944/20117582.471

Este es un artículo de acceso abierto bajo una Licencia Creative Commons - BY-NC-ND https://creativecommons.org/licenses/by-ncnd/4.0/deed.es 


\begin{abstract}
Introduction. Cecal volvulus in pregnancy is an uncommon pathology worldwide, with a varied clinical spectrum and limitation in diagnostic studies, which can result in an ominous outcome.

Clinical case. We present the case of a pregnant woman in the second trimester of pregnancy who presents with an acute abdomen secondary to caecal volvulus and fetal distress. The patient was taken to emergency caesarean section, where a cecectomy and transverse ileus anastomosis were also performed, with fetal mortality.

Conclusion. Cecal volvulus is part of the differential diagnoses in acute abdomen during pregnancy. It has a reserved prognosis, where adverse maternal and perinatal outcomes can occur, including maternal-fetal mortality.
\end{abstract}

Keywords: intestinal volvulus; cecum; acute abdomen; pregnancy; pregnancy complications; pregnancy outcome.

\section{Introducción}

La obstrucción intestinal es una entidad poco frecuente durante el embarazo, con una incidencia que varía entre I en 3600 a I en 60.000 embarazos ${ }^{\mathrm{I}}$. Las principales causas de obstrucción intestinal durante el embarazo incluyen las adherencias intestinales (58\%), vólvulos (24\%) e intususcepción $(5 \%)^{2,3}$. El vólvulo cecal ocurre aproximadamente en I de 50.0000 embarazos e incluso podría ser menor, hasta de I en I.000.000 I, por lo que se considera una patología rara.

Su presentación se basa en un cuadro de dolor abdominal y sintomatología atípica, que dificulta su diferenciación con otras causas de dolor abdominal ${ }^{1,4,5}$. El diagnóstico de esta enfermedad la mayoría de las veces es tardío, ya que la sintomatología causada por la obstrucción intestinal es pasada por alto, generando confusión con cuadros de hiperémesis, ruptura uterina o abruptio de placenta ${ }^{6}$. En una encuesta realizada a gestantes con diagnóstico de vólvulo cecal, se identificó que el $25 \%$ de ellas tuvieron el diagnóstico previo a la cirugía, lo que muestra la dificultad en su manejo ${ }^{2,3}$.

Adicional a esto, se encontró mortalidad materno fetal en un $20-50 \%{ }^{5,78}$ y complicaciones como isquemia intestinal, perforación, peritonitis y choque 9 . Durante el embarazo existen múltiples patologías que pueden requerir la ayuda de imágenes para la confirmación de su diagnóstico ${ }^{1}$, pero el uso inadecuado de estas puede retrasar el tratamiento óptimo ${ }^{\text {Io }}$.

\section{Caso clínico}

Paciente femenina de 2I años de edad, primigestante con embarazo de $294 / 7$ semanas, quien asiste al servicio de urgencias por cuadro clínico de seis horas de evolución consistente en náuseas, vómito y constipación, asociados a dolor abdominal en banda en hemiabdomen superior, irradiado a fosa ilíaca derecha. Refería haber presentado un episodio similar dos semanas antes, el cual había resuelto espontáneamente.

Al examen físico se encontraba taquicárdica, polipneica, afebril, con distensión abdominal y signos de irritación peritoneal dados por dolor al rebote en fosa iliaca derecha. Se practicó una monitoria fetal que mostró bradicardia fetal sostenida como signo de estado fetal no satisfactorio, por lo que fue llevada a cesárea de urgencia encontrando en cavidad peritoneal abundante líquido fétido, color vino oporto. Se obtiene un producto de gestación femenino, peso: $1280 \mathrm{gr}$, APGAR o/I min, o/5 min, BALLARD 29 semanas. Se hizo pinzamiento temprano del cordón umbilical y reanimación neonatal guiada por el servicio de neonatología, que fue fallida.

En la revisión de la cavidad abdominal se identifica isquemia y necrosis en colon ascendente y primera porción de colon transverso, con vólvulo a nivel de la válvula ileocecal (figura I), por lo que se decide llamado intraoperatorio al servicio de cirugía general, quienes proceden a realizar hemicolectomía derecha con anastomosis íleo transversa latero-lateral (figura 2). Se cerró 


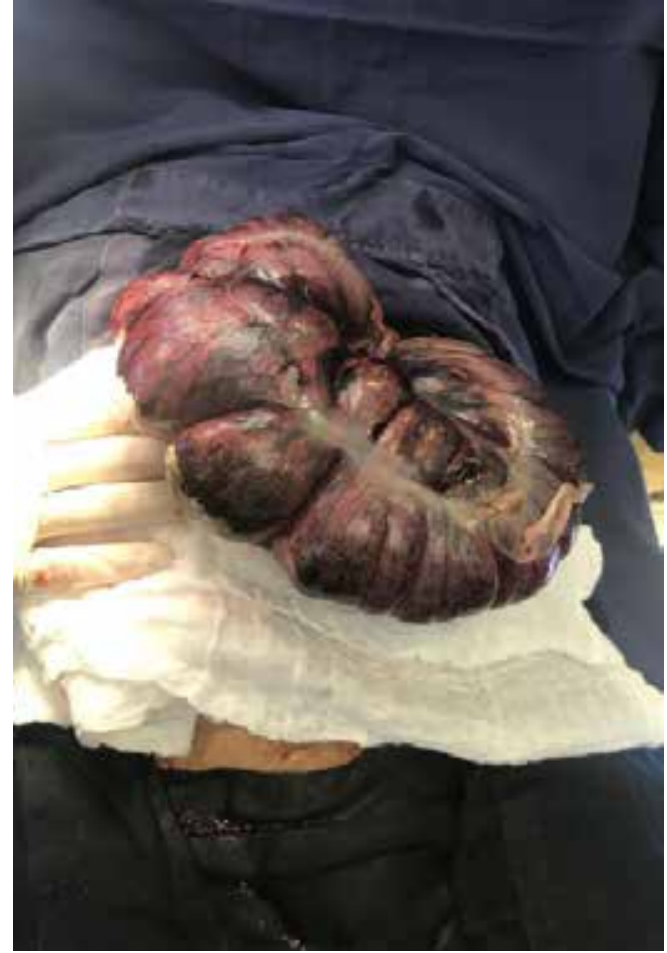

Figura 1. Vólvulo a nivel de la válvula ileocecal.

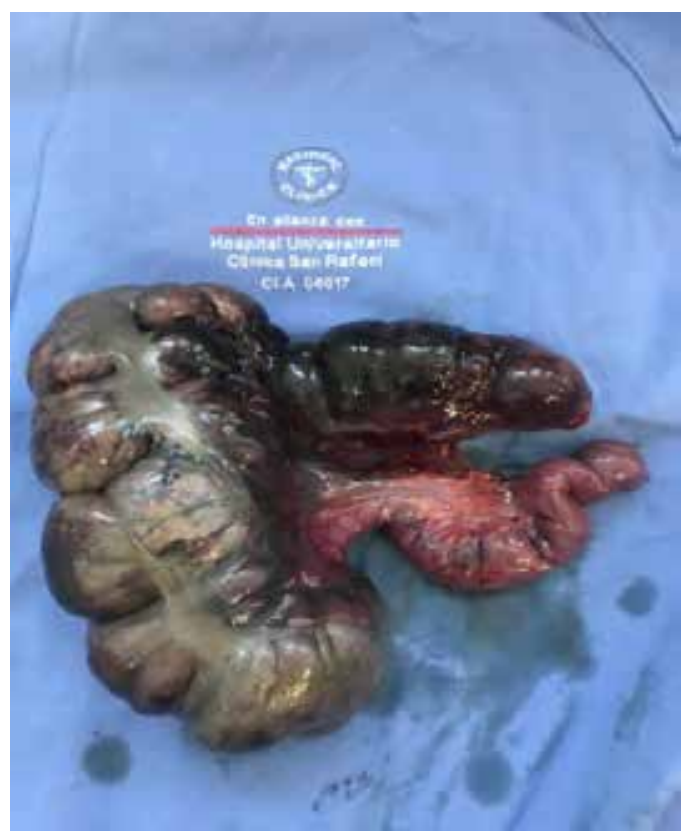

Figura 2. Producto de hemicolectomía derecho donde se aprecia la necrosis del colon. pared abdominal y la paciente fue trasladada a cuidados intensivos para vigilancia y monitorización continua por dos días, sin requerimiento de soporte vasopresor ni nuevas intervenciones quirúrgicas. Se inició vía oral al segundo día postquirúrgico y se trasladó a hospitalización en pisos donde completó vigilancia clínica, manejo antibiótico y terapia por grupo de psicología y psiquiatría por pérdida fetal. Se dio egreso sin complicaciones maternas.

\section{Discusión}

El vólvulo cecal es la torsión del intestino sobre su propio mesenterio, que resulta en una obstrucción de asa cerrada ${ }^{\mathrm{I}}$. Existen diferentes factores de riesgo descritos para el desarrollo de vólvulo cecal como son la dieta, el estreñimiento, uso excesivo de laxantes y diferentes medicamentos ${ }^{\text {II. }}$ Se cree que en la población que presenta hipermovilidad del colon proximal, esto se genera por una mala fijación del peritoneo lateral durante su formación ${ }^{10,12}$. Además, el colon ascendente distal debe estar fijo al retroperitoneo, lo que resulta en un punto de pivote alrededor del cual puede ocurrir la rotación cecal ${ }^{\text {I. }}$.

El embarazo por si solo es un factor predisponente para la aparición de vólvulo cecal, siendo más común en el último trimestre del embarazo, entre la semana 32 y 36 , ya que a medida que va aumentando la edad gestacional, el útero ocupa mayor espacio en la cavidad abdominal, desplazando órganos como el colon, lo que hace que el intestino rote sobre sí mismo ${ }^{13}$. La frecuencia de aparición es mayor en el sigmoides, con un $80 \%$ de los casos, seguido en un $15 \%$ por el ciego y 5 $\%$ en el colon transverso ${ }^{\text {. }}$.

Clásicamente el vólvulo cecal se manifiesta con síntomas de obstrucción intestinal, sin embargo, su presentación clínica muchas veces es imprecisa y puede ser confundida por síntomas frecuentes en el embarazo, como náuseas, vómito y dolor abdominal, haciendo que el diagnóstico sea tardío e irreversible ${ }^{\mathrm{I}}$.

El examen físico es más difícil debido al propio embarazo. Se puede llegar a presentar distensión abdominal, palpación de masa en he- 
miabdomen inferior, incluso signos de irritación peritoneal frente al desarrollo de un cuadro de isquemia mesentérica. Debido a esta sintomatología se puede confundir con otros diagnósticos diferenciales como abruptio de placenta, ruptura uterina, hiperémesis gravídica, torsión ovárica e incluso patologías extrauterinas del embarazo como colelitiasis, colecistitis, apendicitis, infección de vías urinarias o urolitiasis ${ }^{15,16}$.

Respecto al uso de imágenes diagnósticas, existe una dificultad por la restricción en el uso de las mismas en algunos trimestres del embarazo debido a la radiación ionizante. Además, el útero grávido desplaza el posicionamiento de las asas intestinales, por lo que el diagnóstico con imágenes radiológicas puede no ser certero ${ }^{2}$. Dentro de los métodos diagnósticos imagenológicos se incluyen los rayos $\mathrm{X}$, tomografía computarizada y resonancia nuclear magnética. Se debe considerar que la dosis de radiación ionizante permitida en el embarazo es de $5 \mathrm{rad}(50 \mathrm{nGy})^{17,18}$, siendo el riesgo de daño al feto alrededor de i en I0.00o, en especial en el tercer trimestre ${ }^{\mathrm{I}}$. Se considera que la tomografía computarizada es una prueba rápida y segura, con alta precisión diagnóstica en la evaluación del abdomen agudo en la población embarazada ${ }^{13}$.

El uso de la resonancia nuclear magnética como método diagnóstico ha incrementado en las embarazadas, al ser un estudio que utiliza radiación no ionizante ${ }^{\perp}$. Sin embargo, su utilidad en la evaluación del abdomen agudo de la embarazada no está clara y ha sido más indicada en el diagnóstico de apendicitis aguda ${ }^{18,19}$, aunque podría tener una ventaja en el diagnóstico de las obstrucciones intestinales debido a la evidencia de la transición del contraste, identificación de sitios de inflamación, formación de abscesos y sangrado, ya sea de la pelvis o del abdomen ${ }^{20}$.

El tratamiento en la torsión intestinal depende de varios factores, entre ellos, el tiempo del cuadro clínico, la viabilidad del intestino, el número de episodios y la edad gestacional de la paciente. Según estas características, el tratamiento puede ser conservador o quirúrgico.
Independientemente de si la paciente está en embarazo o no, las dos alternativas tienen como objetivo la descompresión intestinal y la prevención de la recurrencia ${ }^{6,9,15}$.

El tratamiento del vólvulo cecal incluye la devolvulación del colon vía colonoscopia, la devolvulación del colon con cecopexia o cecostomía y la hemicolectomía derecha. La colonoscopia se ha empleado para confirmar el diagnóstico y descartar una lesión de la mucosa, evitando la cirugía de emergencia en un proceso agudo, para poder hacer el procedimiento quirúrgico posterior al parto o hasta que el feto sea viable. Sin embargo, el riesgo de recurrencia por este método es mayor a $50 \%{ }^{\mathrm{IO}}$, por lo que no es considerado un tratamiento de primera línea.

Varios estudios retrospectivos sugieren que la recurrencia de vólvulo cecal con devolvulación, cecopexia o cecostomía es inaceptablemente alta; por lo tanto, la hemicolectomía derecha es el tratamiento de elección para esta enfermedad ${ }^{\text {io }}$. Las opciones quirúrgicas dependen del momento de diagnóstico. En el embarazo, la resección de ciego hipermóvil está justificada para evitar la recurrencia y el procedimiento quirúrgico es mucho más seguro antes del último trimestre. Se encuentran diferentes técnicas quirúrgicas como la cecopexia por laparoscopia, la cual es bien aceptada en pacientes que no tiene compromiso de la mucosa, pues presenta bajos índices de complicaciones y recurrencia hasta en un $8 \%{ }^{6}$. Del mismo modo, se propone la resección con ileostomía o con anastomosis, indicada al encontrar isquemia mesentérica; esta presenta grandes beneficios maternos debido a que elimina la probabilidad de recurrencia ${ }^{5,10,21}$. Por esta razón, el mejor tratamiento empleado para el control de síntomas y disminución de la morbi-mortalidad materna es la hemicolectomía derecha con anastomosis ${ }^{\text {II }}$.

Las complicaciones desencadenadas por vólvulo cecal en la paciente embarazada conllevan a potenciales desenlaces como isquemia mesentérica, como ocurrió en nuestro caso, perforación intestinal con peritonitis, choque séptico e hipovolémico, que pueden terminar en muerte ${ }^{9,22}$. La 
mortalidad fetal es secundaria a la disminución del flujo sanguíneo que irriga la placenta por el aumento de la presión intraabdominal ${ }^{23}$.

\section{Conclusiones}

El vólvulo cecal es una entidad infrecuente en las pacientes gestantes. Actualmente su diagnóstico es un reto médico, debido a que se manifiesta con un cuadro clínico inespecífico, por lo cual realizar una buena anamnesis y relacionar la sintomatología con la edad gestacional aumenta el índice de sospecha, permitiendo hacer un diagnóstico temprano de obstrucción intestinal. La isquemia intestinal se desarrolla entre 24 a 72 horas posterior a la torsión, siendo este el tiempo ideal para realizar el diagnóstico y el procedimiento adecuado. El principal objetivo del tratamiento adecuado y oportuno es reducir la mortalidad materno-fetal.

\section{Agradecimientos}

Los autores agradecen a los departamentos de cirugía general y de ginecología y obstetricia del Hospital Universitario Clínica San Rafael, Bogotá, D.C., Colombia, por el apoyo brindado.

\section{Consideraciones éticas}

Consentimiento informado y derecho a la privacidad. Los autores han obtenido el consentimiento informado de los pacientes y/o sujetos referidos en el artículo. Los autores declaran que han seguido los protocolos de su centro de trabajo sobre confidencialidad de los datos.

Conflicto de intereses. Los autores declaran no tener ninguna relación financiera ni personal con otras personas $u$ organizaciones que pudieran dar lugar a conflicto de intereses.

Financiación. Los autores declaran no haber recibido financiación para la realización de este trabajo.

\section{Referencias}

I. Hogan BA, Brown CJ, Brown JA. Cecal volvulus in pregnancy: report of a case and review of the safety and utility of medical diagnostic imaging in the assessment of the acute abdomen during pregnancy. Emerg Radiol. 2008;I5:I27-3I. https://doi.org/I0.IOo7/siol40-007-0642-9

2. Horton PJ, White J, Lake SP. Caecal volvulus and malrotation of the bowel complicating the third trimester of pregnancy. J Obstet Gynaecol. 1997;17:I6o.

https://doi.org/IO.IO80/oI443619750I13735

3. Harer WB Jr, Harer WB Sr. Volvulus complicating pregnancy and the puerperium. Obstet Gynecol. 1958;12:399-406.

4. Atamanalp SS, Kisaoglu A, Ozogul B, Kantarci M, Disci $\mathrm{E}$, Bulut $\mathrm{OH}$, et al. Sigmoid volvulus complicating pregnancy: A case report. Eurasian J Med. 2015;47:75-6. https://doi.org/I0.5I52/eajm.20I4.0I05

5. Narjis Y, Rabbani K, Largab S, Soumani A, Finech B, Dafali AI. Coecal volvulus: An acute complication of pregnancy. J Emerg Trauma Shock. 2010;3:426-7. https://doi.org/I0.4103/0974-2700.7077I .

6. Singla SL, Kadian N, Kadian YS, Goyal A, Sharma U. Caecal volvulus in pregnancy: Is delay in diagnosis avoidable? Asian J Surg. 2005;28:52-4. https://doi.org/IO.IOI6/SioI5-9584(09)60260-8 .

7. Osime OC, Onakewhor J, Irowa OO. Intussusception in pregnancy - A rarely considered diagnosis. African Journal of Reproductive Health. 20I0;I4:I45-8.

8. Aguayo-Macías E, Rodríguez-Valle AJ, Gonzalez-Habib R, Reyes-Sepúlveda H. Vólvulo intestinal en paciente embarazada: reporte de un caso. Ginecol Obstet Mex. 20I2;80:232.

9. Jiménez Rodríguez RM, Díaz Pavón JM, Alarcón del Agua I, Bernardos García C, Álamo Martínez JM, Sousa Vaquero JM. Vólvulo de ciego como causa de obstrucción intestinal. Rev Esp Enferm Dig. 2014;IO0:373-9.

Io. Montes $\mathrm{H}$, Wolf J. Cecal volvulus in pregnancy. Am J Gastroenterol. 1999;94:2554-6.

https://doi.org/IO.IIII/j.I572-024I.1999.01394.x.

II. Rodríguez-Hermosa JI, Martín A, Farrés R, Pont J, Codina-Cazador $\mathrm{A}$, Ruiz $\mathrm{B}$, et al. Obstrucción intestinal por vólvulo de ciego. Cir Esp. 2005;78:385-7. https://doi.org/Io.IoI6/Sooo9-739X(05)70960-X

I2. Moore CJ, Corl FM, Fishman EK. CT of cecal volvulus: unraveling the image. Am J Roentgenol. 2001;177:95-8. https://doi.org/IO.22I4/ajr.I77.I.I770095

I3. Jones D, Wilson J, Warnock N, Alexander DJ. Abdominal pain in pregnancy. BMJ. 2012;17;345:e68I8. https://doi.org/Io.II36/bmj.e68I8

I4. Machado NO, Machado LSM. Sigmoid volvulus complicating pregnancy managed by resection and primary anastomosis: Case report with literature review. Sultan Qaboos Univ Med J. 2009;9:84-8.

I5. Chase D, Sparks D, Dawood M, Perry E. Cecal volvulus in a multiple-gestation pregnancy. Obstetrics \& Gynecology. 2009;1I4(2, Part 2 Suppl):475-7. https://doi.org/IO.IO97/AOG.oboi3e3I8I989578 
I6. John H, Gyr T, Giudici G, Martinoli S, Marx A. Cecal volvulus in pregnancy. Case report and review of the literature. Arch Gynecol Obstet. 1996;258:I6I-4.

https://doi.org/I0.I007/s004040050II9

17. Toppenberg KS, Hill DA, Miller DP. Safety of radiographic imaging during pregnancy. Am Fam Phys. 1999;59:1813-8.

I8. Forsted DH, Kalbhen CL. CT of pregnant women for urinary tract calculi, pulmonary thromboembolism, and acute appendicitis. Am J Roentgenol. 2002;I78:I2856. https://doi.org/Io.22I4/ajr.I78.5.I78I285 .

19. Incesu L, Coskun A, Selcuk MB, Akan H, Sozubir S, Bernay F. Acute appendicitis: MR imaging and sonographic correlation. Am J Roentgenol. 1997;168:669-74. https://doi.org/IO.22I4/ajr.I68.3.90575I2
20. Leyendecker JR, Gorengaut V, Brown JJ. MR imaging of maternal diseases of the abdomen and pelvis during pregnancy and the immediate postpartum period. Radiographics. 2004;24:I30I-I6.

|https://doi.org/IO.II48/rg.245045036

2I. Consorte E, Liu T. Diagnosis and treatment of caecal volvulus. Postgrad Med J. 2005;81:772-6.

https://doi.org/IO.II36/pgmj.2005.0353II .

22. Devanathan M, RajKumar B, Anbarasu S, Kumar M. Cecal volvulus: a case report. Int J Med Sci Public Health. 2015;4:429-32.

23. Aftab Z, Toro A, Abdelaal A, Dasovky M, Gehani S, Abdel Mola A, et al. Endoscopic reduction of a volvulus of the sigmoid colon in pregnancy: case report and a comprehensive review of the literature. World J Emerg Surg. 20I4;9:4I. https://doi.org/IO.II86/I749-7922-9-4I . 